\title{
The influence of external timing cues upon the rhythm of voluntary movements in Parkinson's disease
}

\author{
J S Freeman, F W J Cody, W Schady
}

\begin{abstract}
The ability of patients with Parkinson's disease (PD) and healthy subjects to synchronise finger tapping, produced by rhythmic wrist movements, with auditory signals of target frequencies (range 1-5 Hz) and to sustain such rhythms following sudden withdrawal of auditory cues was studied. Healthy subjects were able, in the presence of auditory cues, to duplicate target frequencies accurately over the range investigated both in terms of mean tapping rate and in regularity of tapping. PD patients were less accurate under these conditions and on average tended to tap too rapidly at the lower (1-3 Hz) target frequencies and too slowly at the highest $(5 \mathrm{~Hz})$ target frequency. In addition, the variability of their tapping rhythms was generally greater. Healthy subjects were able to sustain tapping rhythms well following suppression of auditory signals. By contrast, withdrawal of external timing cues resulted in marked impairment of the patients' rhythm generation. At lower frequency targets (1-3 Hz) patients' tapping rates increased over rates which were already elevated in the presence of external cues. Conversely, at higher target frequencies (4-5 $\mathrm{Hz}$ ), the average tapping rate tended to decline further from previously depressed levels. The accuracy of almost all patients fell outside the normal range. Two patterns of tapping errors were found. The first was hastening of tapping which was most evident at intermediate target frequencies. The second was faltering which occurred mainly at the higher target frequencies. These forms of behaviour may result from inherent abnormalities of internal rhythm generation since they occurred both in the presence and absence of external timing signals. Overall, our findings are consistent with the view that the basal ganglia have a role in the internal cueing of repetitive voluntary movements.
\end{abstract}

\section{(F Neurol Neurosurg Psychiatry 1993;56:1078-1084)}

A current theory of basal ganglia function attributes these motor centres with a primary role in the internal initiation and timekeeping of movements. This view is, in part, founded on the common clinical observation that many patients with Parkinson's disease (PD) experience difficulty in performing repetitive voluntary movements. ${ }^{1}$ An extreme example of this type of movement deficit is the well known "freezing" phenomenon of PD, in which patients exhibit an inability to start or to continue cyclic motor activities such as walking, speech, or handwriting. ${ }^{234}$ In addition, there is much anecdotal evidence that PD freezing may be both triggered and terminated by external sensory inputs. For example, periodic visual inputs from a striped pattern on the floor or a staircase can help PD patients to sustain locomotion. Thus, suppression of freezing by external cues indicates an increased dependence of the rhythm generator upon extrinsic reinforcement for its continued operation. As a corollary, freezing may represent a basic failure of internal rhythm generation by the basal ganglia.

Detailed measurements of the influence of external sensory information on the ability of PD patients accurately to produce rhythmic movements are, therefore, highly relevant in assessing the possible role of the basal ganglia in internal rhythm generation. There is, as yet, a dearth of such quantitative data. Nakamura et al 5678 have reported abnormalities in the ability of PD patients to perform repetitive finger tapping in response to auditory signals and have proposed that characteristic deficits in rhythm formation exist in this disorder.

In the present experiments we have focused upon a quite different aspect of rhythm formation in PD, namely the reliance of patients on external timing cues to generate and maintain rhythms. To this end, we compared the effects of withdrawal of auditory cues upon finger tapping performance in PD patients and healthy subjects. We also investigated whether abnormal rhythm production in PD was related to the occurrence of freezing in everyday activities and to the co-existence of tremor.

\section{Methods}

SUBJECTS

Nine patients (five men, four women) aged $60.8(6.7)$ years, mean (SD), and 12 healthy subjects (five men, seven women) aged $63 \cdot 4$ (7.9) years were studied. All subjects participated with informed consent and the protocols were approved by the local ethical committee.

The diagnosis of PD was made by a consultant neurologist on the basis of the classic 
triad of tremor, rigidity, and bradykinesis and the absence of any atypical signs or symptoms. Patients were investigated while on their routine therapy, which in all cases included standard levodopa formulations and in some cases additional medication. No distinction was made between the "on" and "off" phases. The average duration of the patients' disease at the time of testing was 6.4 (3.0) years. Patients were assessed for rigidity, bradykinesia, and gait, each scored on four point scales $^{9}$ in which $0=$ normal or absent and $3=$ severely disturbed, immediately before experimental sessions. Rigidity: eight patients were graded 1 and one patient graded 2. Bradykinesia: five patients were graded 1 and four patients were graded 2 . Gait: two patients were graded 0 , two patients were graded 1 , three patients were graded 2, and two patients were graded 3. Four of the patients had an asymmetrical pattern of Parkinsonian signs and of these two were more severely affected on the left side and two on the right. Postural tremor was measured accelerometrically at each wrist. Mean peak frequencies for the left and right wrist were, respectively, $6 \cdot 0(1 \cdot 5) \mathrm{Hz}$ and $6 \cdot 1$ (1.5) $\mathrm{Hz}$ with corresponding ranges of $4 \cdot 3-8 \cdot 7 \mathrm{~Hz}$ and $4 \cdot 0-8 \cdot 2 \mathrm{~Hz}$. The incidence of freezing of gait during everyday activities was also scored for individual patients according to a four point scale: 0 , never; 1 , several times per month; 2, several times per week; 3, daily.

\section{FINGER TAPPING TEST}

Subjects sat in a chair with one forearm resting comfortably upon a table placed in front of them. A flexible metal loop was fitted snugly to the index finger just proximal to the terminal interphalangeal joint. The hand was placed palm downwards over a wooden board to which was attached a metal contact plate. Subjects produced tapping of the index finger by making rapid, alternating flexion and extension movements of the wrist. They were instructed to make small but distinct movements, raising the finger about $5 \mathrm{~mm}$ above the contact plate between strikes, so as to minimise any effects of bradykinesia in the patients. Each time the metal loop on the index finger struck the contact plate it completed an electrical circuit and generated a brief voltage pulse.

Auditory cues of target tapping frequencies were played through a loudspeaker as sequences of "clicks". These signals were produced by an electronic signal generator feeding into an audioamplifier. Trains of regularly spaced cue pulses at 1, 2, 3, 4, and 5 $\mathrm{Hz}$ were used. Blocks of trials in which different target frequency cues were presented were interspersed in a pseudorandom manner.

Two main protocols were employed. In protocol 1, tapping performance was recorded over 30 second periods, throughout each of which an auditory cue signal at a given preselected frequency was presented continuously. Subjects were instructed to tap in rhythm with the auditory "clicks". Such recording periods were separated by gaps of about 1 minute to allow subjects to rest. In protocol 2, tapping performance was again recorded over a series of 30 second periods. However, in this case the auditory cues were presented for only the first 10 seconds, after which the "clicks" were abruptly turned off. Subjects were instructed to tap in rhythm with the cue signal during the initial $10 \mathrm{sec}$ ond phase and then to continue to tap at the same rhythm during the remaining 20 second phase of the trial when the cues were absent. Again, 1 minute recovery periods were allowed between trials. Tapping performance of each hand was tested: protocol 1 was first applied to both hands and then both hands were tested with protocol 2 .

\section{DATA ANALYSIS}

A computer was used to sample, store, and analyse tapping and tremor data. Voltage pulse trains corresponding to the occurrence of, respectively, finger taps and auditory cue signals were sampled at $100 \mathrm{~Hz}$ by separate channels of the A/D. Any tap pulse which followed its predecessor by an interval of $<50$ ms was rejected to eliminate counting artefactual contacts. Mean (SD) frequencies and plots of instantaneous frequencies (reciprocals of successive intertap intervals) of tapping pulses were calculated for each sampling period. Accelerometric tremor recordings were sampled at $50 \mathrm{~Hz}$ by one channel of the $\mathrm{A} / \mathrm{D}$ and analysed by Fourier transform to determine the power spectrum, peak frequency and overall power.

\section{STATISTICS}

Multivariate analyses of variance (MANOVA) were applied to test whether overall differences existed between-firstly, the mean rates and variability of tapping performance of the PD and control groups in the presence of auditory cues, and secondly, the tapping performance within each group in the presence and absence of cues. Unpaired $t$ tests (corrected for unequal variance) and paired $t$ tests were used to identify those signal frequencies at which differences existed between the mean tapping rates, respectively, of the PD and control groups and of individual subjects in the presence and absence of cues. These comparisons each involved five sets of dependent tests. In this situation there is an increased risk of false positives (type I errors) and a compensatory $a$ adjustment procedure (for example, Bonferroni) may be applied. In the present analyses, Bonferroni adjustment dictates that a $\mathrm{p}$ value of 0.01 should be interpreted at the $a=0.05$ level of significance. However, many statisticians (see Cohen $^{10}$ for review) consider the Bonferroni adjustment to be excessively conservative and that its use leads to an unacceptable loss of power (increase in type II errors-that is, false negatives). Therefore, precise, nonadjusted $\mathrm{p}$ values are given throughout the text so that readers can decide significance levels. 
Normal

A

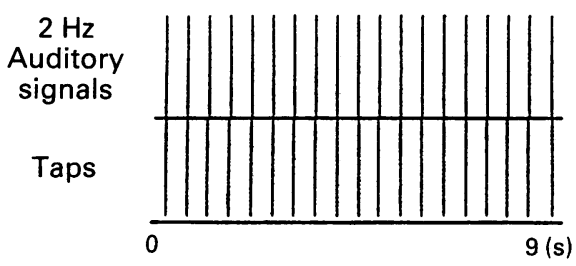

C

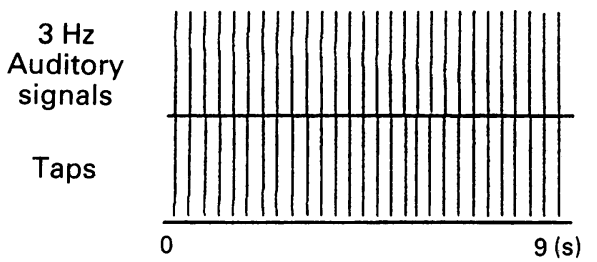

D

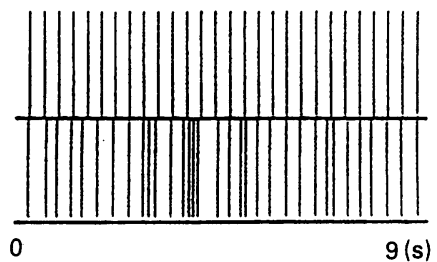

E

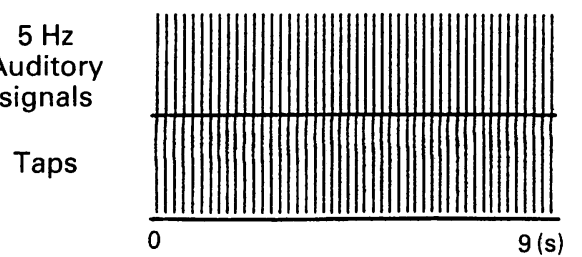

$\mathrm{F}$

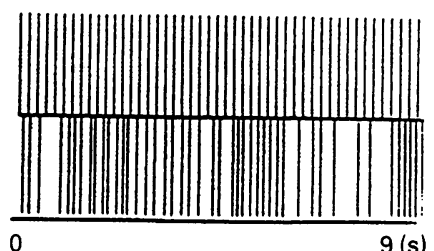

Figure 1 Finger tapping performance of a normal subject and $P D$ patient in the presence of auditory signals of target frequency. Separate series of pulses representing the occurrence of, respectively, auditory signals and finger taps show the relative timing of these two sets of events in 9 second records obtained during 30 second periods of tapping at $2 \mathrm{~Hz}, 3 \mathrm{~Hz}$, and $5 \mathrm{~Hz}$ targets in the normal $(A, C$, and $E)$ and $P D(B, D$, and $F)$ subjects.

\section{Results}

EXTERNALLY SIGNALLED RHYTHM

GENERATION IN PD AND HEALTHY SUBJECTS

Figure 1 compares the abilities of a healthy subject and a representative PD patient to duplicate the rhythm of sequences of auditory cues, presented at varying frequencies, by making voluntary wrist movements to produce tapping of the index finger.

Segments of recordings made from, respectively, the healthy subject (fig 1A, C, and E) and the $P D$ patient (fig $1 B, D$, and $F$ ) during $2 \mathrm{~Hz}, 3 \mathrm{~Hz}$ and $5 \mathrm{~Hz}$ trains of equally spaced auditory "clicks" show characteristic differences in performance. The pattern of pulses produced by the tapping movements of the healthy subject reproduced far more accurately the rhythm of the auditory cues, both regarding mean frequency and regularity, than those of the patient. In particular, the patient tapped too rapidly at lower $(1-3 \mathrm{~Hz})$ signal frequencies and too slowly at higher $(4-5 \mathrm{~Hz})$ signal frequencies. The lack of consistency in the relative timing of tap and cue pulses in figure 1 suggests that neither the PD patient nor the healthy subject responded directly to each individual "click" in the train of auditory signals at any of the cue frequencies. Interspersed in the patient's tapping pulse trains are occasional very short intervals (about 110-125 ms), corresponding to instantaneous tapping rates of $8-9 \mathrm{~Hz}$, which
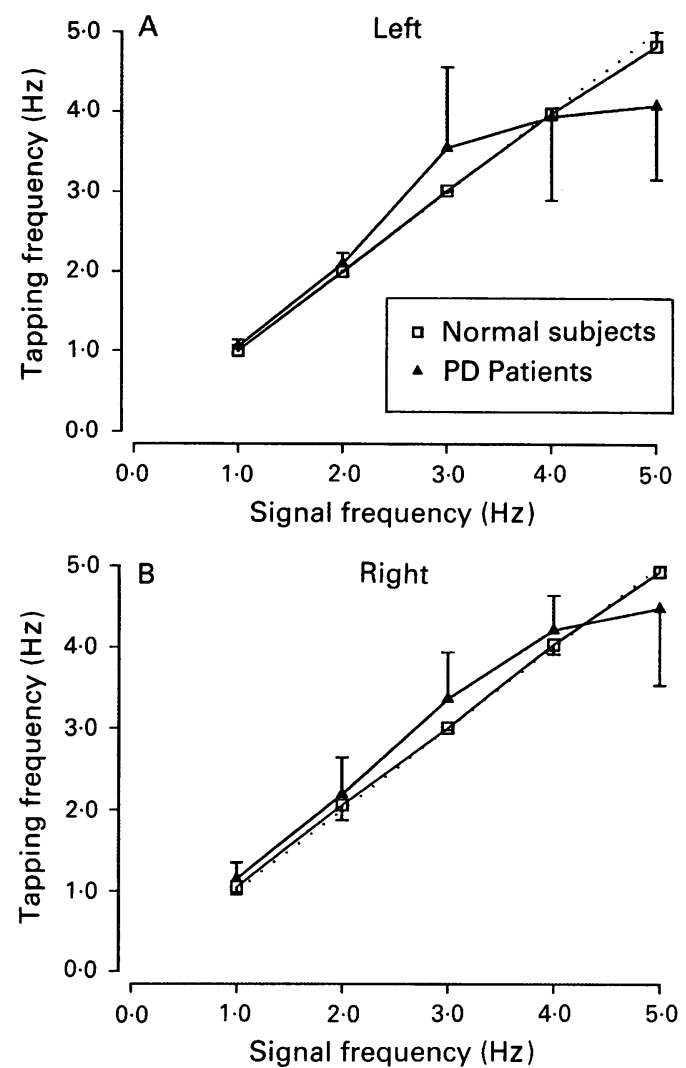

Figure 2 The relationships between finger tapping frequency and the frequency of auditory cue signals in healthy subjects and PD patients. Mean (SD) tapping frequencies are shown. Data for left (non-preferred) hand are plotted in $(A)$ and for the right hand in $(B)$. The dotted line, with unity slope, indicates exact correspondence of tapping and cue frequencies. The mean tapping rates of the PD group, across the range of signal frequencies, differed significantly from those of the control group $(p=0.01, M A N O V A)$.

are not present in the records of the healthy subject. However, equally short intertap intervals appeared in the records of healthy subjects striving to tap at their maximum rate (typically averaging about $6 \mathrm{~Hz}$ for a 10 second period), when their rhythm became far more variable. The mechanism responsible for these sporadic, closely spaced pulse pairs is uncertain. However, none had instantaneous frequencies more than about $10 \mathrm{~Hz}$ and their occurrence had no definite periodicity.

Plots of group data (fig 2) indicate that PD patients were, in general, less exact than healthy subjects in replicating the cue frequency and in producing an even pace. Results from the left (fig 2A; non-preferred in all subjects tested) and right (fig 2B) hands are illustrated. The relatively larger SD values of the patient group suggest that the tapping performance of the patients, at each signal frequency, was more variable than that of the controls and this was confirmed by statistical analysis ( $p=0.01$, MANOVA, note that nonBonferroni adjusted $p$ values are given throughout, see Methods). The mean tapping rates, considered across the overall range of target frequencies, of the patient and control groups also differed ( $p=0.01$, MANOVA). Figure 2 shows that the PD patients as a whole tended to tap more rapidly than healthy subjects at low intermediate signal 

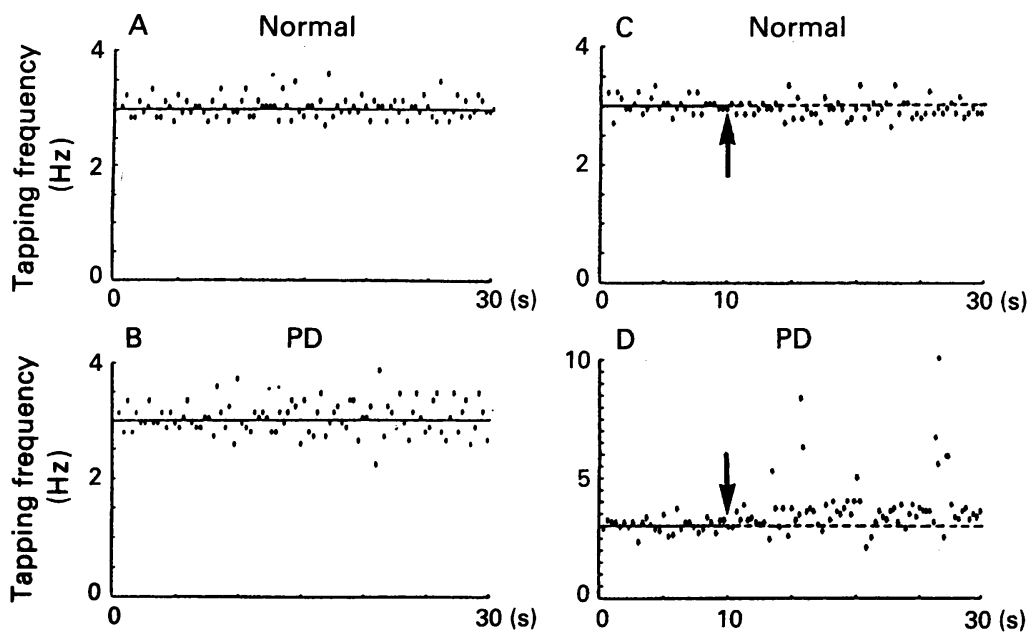

Figure 3 Plots of instantaneous frequency of finger tapping in a healthy subject and PD patient in the continuous presence of $3 \mathrm{~Hz}$ auditory signals and following removal of cues. Each point represents the occurrence of a single tap. The height of each point indicates its instantaneous frequency in relation to the immediately preceding tap and was calculated as the reciprocal of the intertap interval. In $(A)$ and $(B)$ the tapping performances of, respectively, the healthy subject and $P D$ patient in the continuous presence of cues are shown. The solid horizontal lines indicate cue frequency. In (C) and (D) the auditory signal was played, respectively, to the healthy subject and patient during the first 10 s of each plot. At the times indicated by the arrows the auditory signals were turned off and tapping during the remaining 20 s periods was in the absence of auditory cues. The solid horizontal lines in the initial 10 second periods of the records show the frequency of the auditory signals. These levels are continued as dashed lines in the later 20 second periods. The scales of the vertical axes differ in (D) from those in $(A)(B)$ and $(C)$.

frequencies and less rapidly at the highest (5 $\mathrm{Hz}$ ) frequency investigated. The mean left hand tapping rates of the patient group were higher than those of the control group for cue frequencies of $1 \mathrm{~Hz}$ and $2 \mathrm{~Hz}$ and lower for the $5 \mathrm{~Hz}$ signals ( $\mathrm{p}$ values, respectively, $0.004,0.009$, and $0.036, t$ test). Essentially similar findings were obtained for right (preferred) hand performance, although only at 3 $\mathrm{Hz}$ did the difference approach a significant level $(p=0.049)$. In neither the patient nor control groups were there significant differences between the mean tapping rates of left versus right hands at any of the target frequencies.

Detailed investigation of the behaviour of individual patients revealed that two distinct subclasses of tapping abnormalities contributed to the impaired accuracy, in terms of mean rates, of the PD group as a whole. Abnormal performance was defined as tapping rates which fell, at two or more target frequencies, outside the range of values obtained from healthy subjects. Data for each Parkinsonian hand (18 hands from nine patients) were analysed independently. The first pattern of abnormality, termed hastening, comprised an increase and the second, termed faltering, comprised a reduction in mean tapping frequencies. Instances of hastening (four hands) were most common at $3 \mathrm{~Hz}$ and $4 \mathrm{~Hz}$ targets while faltering (four hands) was only displayed at the higher $(4 \mathrm{~Hz}$ and $5 \mathrm{~Hz}$ ) target frequencies. At the $5 \mathrm{~Hz}$ target, some hasteners tapped in excess of the signal frequency. Only one patient showed abnormal performance with both hands and she displayed contrasting patterns on the two sides.
RHYTHM GENERATION IN THE ABSENCE OF EXTERNAL SIGNALS IN PD AND HEALTHY SUBJECTS

Figure 3 presents instantaneous frequency plots of the tapping performance of representative healthy and PD individuals during 30 second periods in which a $3 \mathrm{~Hz}$ auditory cue signal was either present throughout ( $A$ and B) or removed after 10 seconds (C and D).

The plots obtained during continuous auditory signals show two main features. Firstly, the healthy subject (fig 3A) and PD patient (fig 3B) were both able to establish almost immediately a tapping rhythm approximating to the cue frequency upon onset of the auditory signal. Secondly, in neither case is there any definite sign of a transition in performance such as might result from loss of concentration or tiredness. Comparison of the tapping performance of individual subjects during the first and last 10 second periods of the 30 second tests confirmed that in neither patients nor controls was there a systematic deterioration in accuracy as trials progressed. Similar findings were obtained for all target frequencies.

Figure $3 \mathrm{C}$ shows that the tapping performance of the healthy subject was relatively unaffected by withdrawal of the auditory signals. By contrast, the performance of the patient (fig $3 \mathrm{D}$, note different frequency scale) underwent a clear alteration following removal of auditory cues. There was an increase in average tapping frequency and the rhythm became far more irregular. Similar trends were noted for the $3 \mathrm{~Hz}$ target frequency in the patient group as a whole (see below). These changes seem certain to have resulted from withdrawal of cue signals, since they did not occur in the presence of auditory cues (fig 3B).

The plots in figure 4 compare the tapping performance of healthy and PD groups in the presence of auditory signals and following their sudden removal once the rhythm had been well established. Results for the left (non preferred) hand are illustrated.

Figure 4 shows that the withdrawal of external cues had a pronounced influence on the performance of the patients (fig 4B) whereas it had little effect on that of the healthy subjects (fig 4A). Statistical analysis confirmed that the mean tapping rates of the PD group, across the whole range of signal frequencies, differed significantly in the presence and absence of cues ( $p=0.01$, MANOVA) while those of the control group did not. Cue suppression resulted in the patients' mean tapping frequencies increasing at the two lowest target frequencies $(1 \mathrm{~Hz}$ and $2 \mathrm{~Hz}$ ) and declining at the $4 \mathrm{~Hz}$ target $(\mathrm{p}$ values, respectively, $0.038,0.027$, and 0.033 , paired $t$ test). Similar findings were obtained for their right (preferred) hand tapping performance. Withdrawal of cue signals caused increases in mean tapping rates at targets of 1,2 , and $3 \mathrm{~Hz}$ and a reduction at $5 \mathrm{~Hz}$ (p values, respectively, $0.009,0.035,0.028$, and 0.047 , paired $t$ test). In all cases, these changes represented a further reduction in 
Figure 4 Plots comparing the tapping performance of subjects in the presence and absence of auditory signals for the group of healthy subjects $(A)$ and group of $P D$ patients $(B)$. Mean (SD) values for the left (non-preferred) hand are given. Dotted lines indicate unity slope. The mean tapping rates of the $P D$ group, across the range of signal frequencies, differed significantly in the presence and absence of auditory cues $(p=0.01$. $M A N O V A)$. Those of the control group did not.
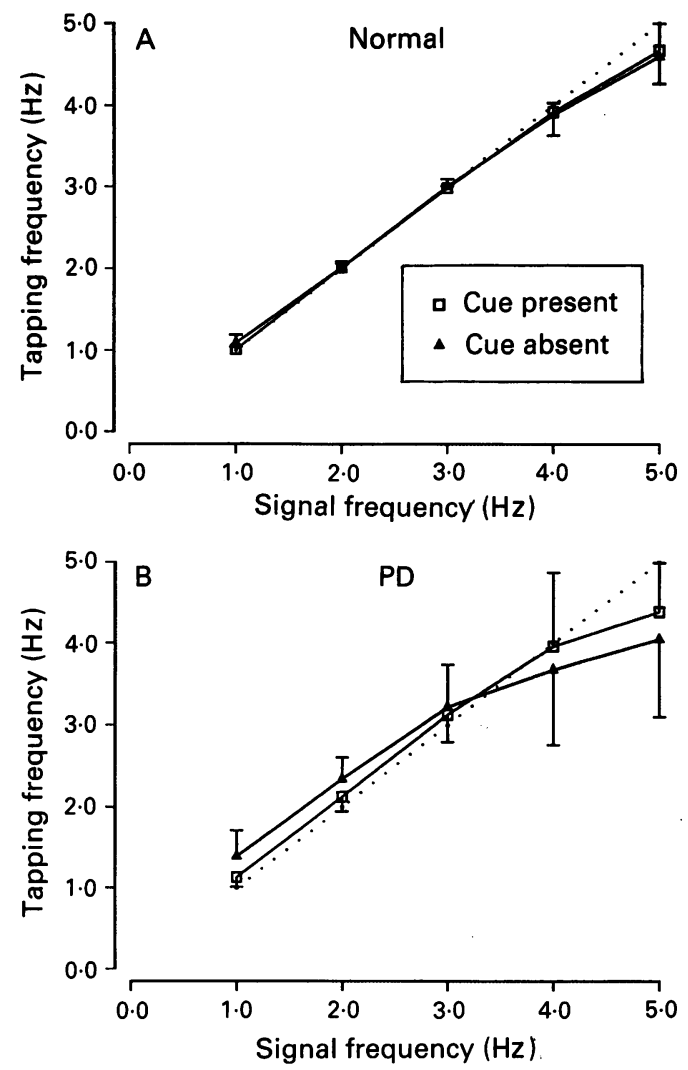

accuracy. Although these effects of cue suppression, at individual signal frequencies, are often at borderline levels of statistical significance (see Methods), the fact that they were observed bilaterally strongly supports their general validity.

Analysis of the performance of individual patients' hands demonstrated that instances of the two subclasses of abnormal performance, hastening and faltering, were found following cue withdrawal. Hastening was now the predominant pattern (six hands out of 18 possible instances) and was most commonly noted for $2-4 \mathrm{~Hz}$ targets while faltering (two hands) was observed at 3-5 $\mathrm{Hz}$ targets.

\section{RELATIONS OF ABNORMAL RHYTHM}

GENERATION IN PD TO TREMOR, BRADYKINESIA, AND FREEZING

Tremor characteristics were compared between PD hands showing different types of tapping abnormalities. PD hands were grouped according to hastening, faltering, or "within normal" behaviour as defined above. Neither the mean peak frequency nor the power of postural tremor differed significantly ( $t$ test) between these groups. Individual instances of abnormal tapping performance were noted in the absence of appreciable tremor, while in other instances tapping accuracy was "within normal limits" despite the presence of pronounced, low frequency tremor.

To analyse the relation of abnormal tapping performance to bradykinesia and freezing, patients were categorised as hasteners or falterers depending on whether they displayed exclusively hastening or faltering performance as previously defined. Two patients were excluded: one showed different abnormalities in the two hands and one exhibited an alteration in pattern between the test conditions. There was no evidence of any differences between these two patient categories concerning scores of clinical bradykinesia. However, there was a tendency for the patients in the hastener group to show more frequent freezing episodes. All four of these patients had freezing ratings of 2 or 3, whereas each of the three patients in the falterer group had ratings of 0 or 1 .

\section{Discussion}

The present findings demonstrate that the ability of PD patients to generate simple, rhythmic voluntary movements is impaired in two distinct respects. First, and in agreement with previous studies, ${ }^{567}$ patients are less able than healthy subjects to synchronise accurately their movements to extrinsic timing cues. Secondly, the patients exhibit a greater reliance on external cues for rhythm formation. Thus, in PD there appears to be a deficit of external guidance co-existing with a probably more fundamental derangement of internal cueing for stereotyped, repetitive voluntary movements.

\section{ABNORMALITIES OF SYNCHRONISATION OF} MOVEMENTS TO EXTERNAL CUES IN PD

Our findings generally confirm earlier observations of Nakamura and colleagues ${ }^{5-7}$ for finger tapping and of Logigian et $a l^{11}$ for repetitive isometric contractions of finger muscles that PD patients show two separate types of synchronisation abnormalities. The first is an elevation of tempo as targets exceed $2-3 \mathrm{~Hz}$ and the second a depression of tempo at targets approaching $5 \mathrm{~Hz}$.

A straightforward explanation of the latter form of behaviour, since slowness of movement is a common feature of PD, is that patients cannot produce sufficiently rapid movements. We cannot exclude the possibility that an inherent inability of some of our patients to maintain an adequate pace of movement contributed to their synchronisation errors for the $5 \mathrm{~Hz}$ target. However, a number of patients with extreme disturbances of rhythm formation, the hasteners, actually achieved mean tapping rates in excess of 5 Hz. Thus, slowness of movement was not a universal limiting factor in the PD group and a more basic synchronisation deficit was probably responsible.

This view is supported by the occurrence in patients' records of sporadic groups of two to three taps at instantaneous frequencies of 8-9 Hz. Such closely spaced taps, which contribute to the irregularity of rhythms in PD, are unlikely to have been produced by individually planned, separate movements. The fact that none occurred at instantaneous rates exceeding about $10 \mathrm{~Hz}$ suggests that they were not simply artefactual contacts; if so, rates up to $20 \mathrm{~Hz}$ (for $50 \mathrm{~ms}$ rejection interval) would be anticipated. Although an interaction between Parkinsonian tremor and 
voluntary activity has been reported, ${ }^{11}$ the lack of any obvious periodicity in the occurrence of these high frequency bursts argues against such a mechanism having been the sole cause. A clue to their likely origin is provided by the appearance of similar $8-9 \mathrm{~Hz}$ pulse pairs in the records of healthy subjects attempting to tap at maximal rate when intertap variability increases dramatically and approaches Parkinsonian levels. Present mod$\mathrm{els}^{12}$ of repetitive movement propose that a central neural oscillator sets the tempo of the rhythm. Thus, the increased variability of tapping of healthy subjects at maximal rates may be due to destabilisation of such a neural oscillator. If so, the occurrence of an analagous oscillator instability, but at relatively lower frequencies, may underlie PD patients' difficulties in synthesising sequences of regular and appropriately paced movements.

DEPENDENCE OF PD PATIENTS ON EXTERNAL CUES FOR RHYTHM FORMATION

The main new finding of the present study is that the tapping performance of PD patients deteriorates following withdrawal of external timing cues. Tapping rates increased for low intermediate frequencies and decreased for high target frequencies. These changes correspond to an exaggeration of pre-existing patterns of inaccuracies found in the presence of timing cues.

Models of rhythmic movement generation by the nervous system comprise two conceptually distinct elements, namely an internal timekeeper or oscillator component and an implementation or effector component. ${ }^{13}$ Although movement implementation itself is abnormal in PD, inaccuracies of repetitive movement arising from this source are likely to occur equally in both the presence and absence of external timing cues. The increased dependence of $P D$ patients on external timing cues for rhythm formation, therefore, argues strongly in favour of a deficit of internal timekeeping. Since healthy subjects are able to generate motor programmes for ongoing repetitive movements by extrapolating temporal parameters from previous similar movements, ${ }^{14}$ this capacity must be assumed to be impaired in PD.

Nakamura et $a l^{5-7}$ have, on quite separate grounds, also proposed a disturbance of internal rhythm formation in PD. These authors placed considerable emphasis on a hastening phenomenon in PD which was characterised by a clear cut transition frequency of $2-3 \mathrm{~Hz}$, at which the tapping rate suddenly jumped to a new plateau level of 5-6 Hz. The latter frequency was thought to represent an intrinsic oscillation occurring within the healthy nervous system which is unmasked in PD. In this respect, however, the present results differ from those of Nakamura et al. ${ }^{5-7}$ The tapping rhythms of our PD patients very rarely showed an obvious transition frequency or any sign of convergence to a single frequency.
RELATION OF ABNORMALITIES OF RHYTHM GENERATION TO TREMOR, BRADYKINESIA, AND FREEZING IN PD

Tremor is a common feature of PD. It occurs predominantly at frequencies of $4-6 \mathrm{~Hz}^{15}$ and is believed to result from oscillatory discharge of neurons in the ventrolateral thalamus. ${ }^{1617}$ Logigian et $a l^{11}$ have proposed that in PD the neural oscillators for repetitive voluntary movement and for tremor become synchronised. The general tendency of our patients' tapping frequency to shift towards the PD tremor band is consistent with this "attractor" theory. However, none of the patients showed a dominant frequency in their tapping behaviour such as would be expected if a powerful entrainment occurred. More importantly, the incidence of the different types of abnormal tapping performance (hastening or faltering) among our PD sample correlated with neither the peak frequency nor the power of the recorded postural tremor. In addition, clear instances of tapping deficits were seen in patients lacking appreciable tremor. This echoes the finding of Narabavashi and Nakamura ${ }^{3}$ that disturbances of repetitive tapping persist in PD patients following alleviation of tremor (and rigidity and akinesia) by medication or surgical thalamotomy. Therefore, while the tapping performance of our patients was presumably influenced to some extent by coexisting tremor (and its pathological neural generator), the balance of evidence favours a more fundamental disruption of an independent internal oscillator for repetitive voluntary movement.

As discussed earlier, slowness of voluntary movement does not appear to be responsible for inaccuracies of rhythmic tapping movements in PD. Equally, there was no systematic association between the presence of hastening or faltering tapping behaviour and the clinical score of bradykinesia.

Nakamura et $a l^{36}$ regarded the hastening phenomenon they observed in rhythmic finger tapping as closely related to PD freezing. A relationship between hastening and freezing is also suggested by the observation that PD patients often show a freeze-release-hastenfreeze cycle gait and speech in which, for example, freezing episodes during walking are immediately preceded by a quickening of pace and shortening of step. ${ }^{18}$ In keeping with this notion, we found that all of our PD patients whose sole tapping disorder was an abnormally increased rate reported relatively high incidences of freezing episodes during everyday activities. Therefore, some common pathological processes probably predispose to disturbances of rhythmic hand movements and freezing in PD, although it would be premature to assume a single causative mechanism.

Overall, the present finding that our patients were abnormally dependent upon external timing cues to regulate the tempo of finger tapping indicates that in PD deficits of central programming are not confined to the sequencing of relatively complex movements 
(see Refs 19-22) but also apply to the rhythm generation which underlies extremely simple, stereotyped, and repetitive voluntary movements.

The work was supported by the MRC. JSF was in receipt of a SERC Studentship. We wish thank Ms Frances Culshaw for development of computer software and tapping apparatus and Drs RG Lascelles and D Neary for allowing us to study patients under their care.

1 Marsden CD. The mysterious motor function of the basal ganglia: the Robert Wartenberg lecture. Neurology 1982; 32:514-39.

2 Andrews CJ. Influence of dystonia on the response to long-term L-dopa therapy in Parkinson's disease. $f$ Neurol Neurosurg Psychiatry 1973;36:630-6.

3 Narabayashi H, Nakamura R. Clinical neurophysiology of freezing in parkinsonism. In: Delwaide PJ, Agnoli A, eds. Clinical neurophysiology in Parkinsonism. New York: Elsevier, 1985:49-57.

4 Kanazawa I. Clinical pathophysiology of basal ganglia disease. In: Vinken PJ, Bruyn GW, Kiawans HI, eds. Handbook of clinical neurology. Vol 5. Extrapyramidal disHandbook of clinical neurology. Vol 5. Extrat
orders. New York: Elsevier, 1986:65-91.

5 Nakamura R, Nagasaki H, Narabayashi H. Arrhythmokinesia in parkinsonism. In: Birkmayer W, Hornykiewicz O, eds. Advances in Parkinsonism. Basle: Roche, 1976:258-68.

6 Nakamura R, Nagasaki H, Narabayashi H. Disturbances of rhythm formation in patients with Parkinson's disease: Part I. Characteristics of tapping responses to the periodic signals. Percept Mot Skills 1978;46:63-75.

7 Nagasaki $H$, Nakamura $R$, Taniguchi $R$. Disturbances of rhythm formation in patients with Parkinson's disease: Part II. A forced oscillation model. Percept Mot Skills Part II. A forced

8 Nagasaki H, Nakamura R. Rhythm formation and its dis- turbances: a study based upon periodic response of a motor output system. F Hum Ergology 1982;11:127-42.

9 Webster DD. Critical analysis of the disability in Parkinson's disease. Modern Treatment 1968;5:257-82.

10 Cohen J. Things I have learned (so far). Am Psychol 1990;45:1304-12.

11 Logigian E, Hefter H, Reiners K, Freund H-J. Does tremor pace repetitive voluntary behavior in Parkinson's disease? Ann Neurol 1991;30:172-9.

12 von $G$ alen $G$, Wing $A$. In: Smith $M$, Wing $A$, eds. The psychology of human movement, London: Academic Press, 1984:135-51.

13 Wing A, Kristofferson A. Response delays and the timing of discrete motor responses. Percept Psychophys 1973, 14:5-12.

14 Schmidt RA, Zelaznick HN, Hawkins B, Frank JS, Quinn JT. Motor output variability: a theory for the accuracy of rapid motor acts. Psychol Rev 1979;86:415-51.

15 Findley LJ, Gresty MA, Halmagyi GM. Tremor, the cogwheel phenomenon and clonus in Parkinson's disease. $f$ Neurol Neurosurg Psychiatry 1981;44:534-46.

16 Alberts W. A simple view of parkinsonian tremor Electrical stimulation of cortex adjacent to the rolandic fissure in awake man. Brain Res 1972;44:357-69.

17 Lee R, Stein RB. Resting tremor reset by mechanical perturbations: a comparison of essential and Parkinsonian turbations: a comparison of essential

18 Ward CD. In: Swash M, Oxbury J, eds. Clinical neurology. Edinburgh: Churchill Livingstone, 1991:1395-474.

19 Flowers KA. Lack of prediction in the motor behaviour of parkinsonism. Brain 1978;101:35-52.

20 Benecke R, Rothwell JC, Dick JPR, Day BL, Marsden $\mathrm{CD}$. Simple and complex movements off and on treatment in patients with Parkinson's disease. $\mathcal{f}$ Neuro Neurosurg Psychiatry 1987;50:296-303.

21 Stelmach GE, Teulings H-L. Temporal and spatial characteristics of repetitive movement. Int $\mathcal{f}$ Neurosci 1987 35:51-8.

22 Harrington DL, Haaland KY. Sequencing in Parkinson's disease: abnormalities in programming and controlling movement. Brain 1991;114:99-115.

\section{Neurological stamp}

\section{Luigi Galvani (1737-98)}

Luigi Galvani, the anatomist, physician and physiologist who discovered 'animal electricity', came from Bologna. The galvanometer, which was invented by Andre Ampère (1775-1836), was named after Galvani as was the process of covering steel with a layer of zinc (galvanism).

Galvani observed that static electricity that was stored in a Leyden jar caused dissected frogs' legs to twitch. This occurred if they were placed on metal during a thunderstorm. He also noted that when dissected frogs' legs were hung from brass hooks on an iron railing, the muscles contracted when they came into contact with the iron. Galvani concluded that the source of the electricity was in the muscles and nerves of the animals. His findings were later disproved by Alessandro Volta who by 1800 had constructed electric batteries consisting of two different metals in an electrolytic salt solution. Volta established that the source of the electricity in Galvani's experiment had been two different metals with the animals' body fluids acting as the conducting medium. Galvani's observations were, however, the starting point of electrophysiology.

Galvani was honoured with this Italian stamp in 1934 on the occasion of the First International Congress of Electro-Radio-Biology (Stanley Gibbons 423, Scott 330).

L F HAAS

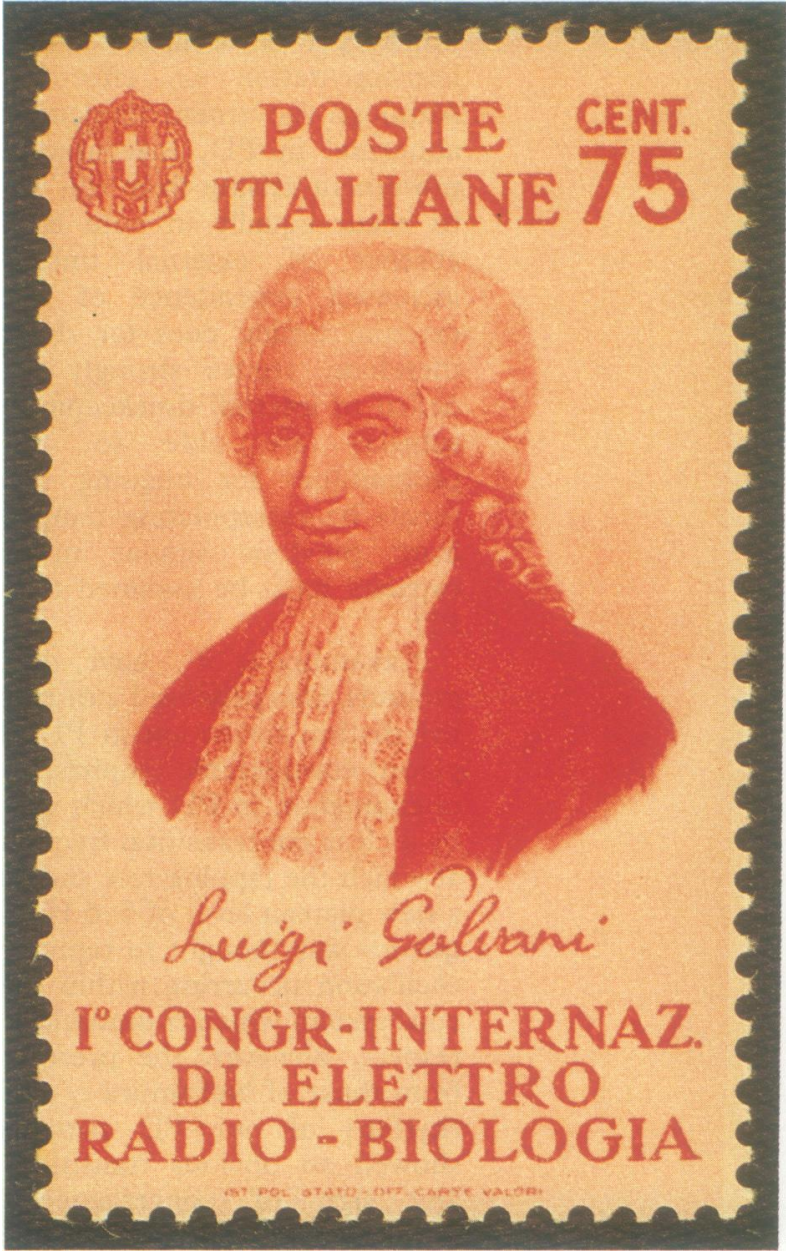

